\title{
Daniel Defoe, un dissident au service de l'orthodoxie protestante
}

Daniel Defoe, a Dissenter Vindicating Protestant Orthodoxy

\section{Yannick Deschamps}

\section{(2) OpenEdition}

1 Journals

\section{Édition électronique}

URL : http://journals.openedition.org/rfcb/3536

DOI : $10.4000 /$ rfcb.3536

ISSN : 2429-4373

Éditeur

CRECIB - Centre de recherche et d'études en civilisation britannique

\section{Édition imprimée}

Date de publication : 1 mars 2013

ISBN : 2-911580-37-0

ISSN : 0248-9015

\section{Référence électronique}

Yannick Deschamps, « Daniel Defoe, un dissident au service de l'orthodoxie protestante », Revue Française de Civilisation Britannique [En ligne], XVIII-1 | 2013, mis en ligne le 01 mars 2013, consulté le 22 mars 2020. URL : http://journals.openedition.org/rfcb/3536; DOl : https://doi.org/10.4000/rfcb. 3536

Ce document a été généré automatiquement le 22 mars 2020.

\section{cc) (1)}

Revue française de civilisation britannique est mis à disposition selon les termes de la licence Creative Commons Attribution - Pas d'Utilisation Commerciale - Pas de Modification 4.0 International. 


\title{
Daniel Defoe, un dissident au service de l'orthodoxie protestante
}

\author{
Daniel Defoe, a Dissenter Vindicating Protestant Orthodoxy
}

Yannick Deschamps

1 Dans un article intitulé 'Defoe's Religious Sect', John Robert More cherche à déterminer la confession - ou la "secte ", selon l'expression utilisée par Voltaire dans ses Lettres philosophiques (1734) ${ }^{1}$ - de Daniel Defoe. Ce dernier est-il quaker, baptiste, indépendant ou presbytérien? Après avoir envisagé avec sérieux chacune de ces hypothèses, More se prononce clairement en faveur de la dernière : Defoe est presbytérien, point de vue qui fait aujourd'hui l'objet d'un consensus. More prend à juste titre pour acquis que Defoe est un dissident protestant, en d'autres termes que, nonobstant son obédience protestante, il se situe en marge de l'Église établie d'Angleterre ${ }^{2}$. De fait, s'il est discret sur son presbytérianisme, Defoe, dans ses nombreux écrits, fait souvent allusion à son statut de dissident. Il le revendique, et se fait même volontiers le porte-parole de ses coreligionnaires ${ }^{3}$. Il remplit ce rôle avec constance jusqu'à la fin du règne d'Anne (1702-1714), pendant lequel la " haute Église » (high Church) est très active, et constitue une menace sérieuse pour les dissidents. Par la suite, il tend toutefois à le délaisser pour endosser celui de défenseur de l'orthodoxie protestante. Ceci ne signifie pas que Defoe s'éloigne de ses racines dissidentes, ni qu'il fait allégeance à l'Église établie. Celleci ne possède pas à ses yeux le monopole de l'orthodoxie. Cependant, les dissidents n'en sont pas non plus les uniques détenteurs. Si l'Église anglicane et les Églises dissidentes comptent de nombreux fidèles dont la foi est conforme à l'orthodoxie protestante, elles abritent chacune en leur sein des brebis égarées coupables d'hétérodoxie. Le déisme, l'arianisme, et même l'athéisme détournent un nombre toujours plus grand de fidèles de la foi protestante authentique. Defoe met donc le contentieux entre anglicans et dissidents sous le boisseau. L'heure est à l'union des protestants face à la montée des croyances hétérodoxes. C'est à l'intention de tous les protestants, et non de ses seuls coreligionnaires dissidents, qu'il rédige ses manuels d'instruction religieuse The Family Instructor (1715) et The New Family Instructor (1727) : 
In the pursuit of this Book [The Family Instructor] care is taken to avoid Distinctions of Opinion, as to Church of England or Dissenter, and no Offence can be taken here either on the one side or the other; as I hope both are Christians, so both are treated here as such, and the Advice is impartially directed to both without the least Distinction. ${ }^{4}$

2 Dans The Family Instructor, Defoe expose sa vision du protestantisme. Dans The New Family Instructor, il s'applique à discréditer les croyances qui menacent la foi protestante: non seulement le catholicisme et le judaïsme, mais surtout le déisme, l'arianisme et l'athéisme. Il s'attelle également à cette tâche dans son traité démonologique, The Political History of the Devil (1726). Même si, dans The New Family Instructor, Defoe consacre davantage de pages à pourfendre l'adversaire catholique traditionnel, sa principale cible est sans conteste l'arianisme, sur lequel se clôt l'ouvrage. De fait, c'est parmi les dissidents et, plus encore, parmi les presbytériens - la propre famille religieuse de Defoe - que cette hérésie connaît le plus vif succès ${ }^{5}$. Confronté à la division grandissante de ses coreligionnaires entre orthodoxes et antitrinitaires, ou "rationnels ", Defoe se doit d'intervenir. Il le fait à une période cruciale de l'histoire des dissidents et des presbytériens; alors que se met en place un processus qui, à terme, conduira leur mouvement vers l'unitarisme ${ }^{6}$. Nous verrons tout d'abord quels sont les principaux principes protestants défendus par Defoe, et quelle est sa conception de l'orthodoxie. Nous nous pencherons ensuite sur son réquisitoire contre les croyances hétérodoxes. Ce faisant, nous constaterons que la ligne de démarcation entre orthodoxie et hétérodoxie telle qu'elle est établie par Defoe ne recoupe en aucune manière le clivage entre anglicans et dissidents. Enfin, nous montrerons que la conception de l'orthodoxie défendue par Defoe peut s'expliquer par son attachement à une forme de presbytérianisme associée à Samuel Annesley.

\section{Une profession de foi protestante}

3 Dans son premier manuel d'instruction religieuse, The Family Instructor (1715), Defoe expose les grands principes qui guident sa foi protestante. Ceux-ci sont énoncés, entre autres, au cours d'un dialogue entre un père, dont le nom n'est pas donné, et son jeune fils Thomas. Le père commence par affirmer l'existence de Dieu, dont il énumère les attributs: 'God is ONE, infinite, eternal, incomprehensible, invisible BEING, the first Cause of all things; the Giver of Life and Being to all things; existing prior, and therefore superior to all things, infinitely perfect, great, holy, just, wise, and good'7. Il insiste sur le fait que Dieu est le créateur de toute chose et, par conséquent, de Tommy. Est ensuite traitée la question du péché originel. Tommy, au même titre que l'ensemble de l'humanité, est responsable de la faute commise par Adam et Ève ${ }^{8}$. Il est corrompu et naturellement enclin à faire le mal, comme il le reconnaît d'ailleurs lui-même : 'I begin to understand [...] That I am born with a Wicked Heart' ${ }^{\prime}$. De la doctrine du péché originel, le père de Tommy passe à celle de la rédemption. Ce faisant, il évoque les mystères de l'Incarnation et de la Résurrection, et souligne la divinité du Christ et sa consubstantialité avec le Père : 'Jesus Christ is essentially God, tho' in a second Person; he is God co-equal, co-eternal, that is, the same in Being, Nature and Attributes; God manifested in the flesh, sent from Heaven to redeem a lost World' 10. Il aborde ensuite le rôle de l'Esprit-Saint, ce qui le conduit naturellement à la doctrine de la Trinité : 'The Godhead is received and understood by us in Three Persons, the Father, the Son, and the Spirit, and these Three are one God, the Maker and Judge of all' ${ }^{11}$. Par ailleurs, le père de Tommy accorde une place importante aux notions d'élection, de 
réprobation et de grâce, et défend sans réserve le dogme de la prédestination : 'We cannot say all are Saved [...] all those who are Saved, are so Saved [...] by the Satisfaction of the Blessed Redeemer, being chosen from Eternity by the meer Grace and Good-will of God'12. Cette leçon de catéchisme inclut également la doctrine de la conversion spirituelle, ou régénération. Indispensable au salut, celle-ci est accordée par la grâce divine aux seuls élus ${ }^{13}$.

En soulignant, dans sa défense de la Trinité, l'égalité et la consubstantialité entre le Père et le Fils, Defoe dénonce en creux l'arianisme, selon lequel le Fils est subordonné au Père, qui l'a créé. De fait, ce mouvement né au IV siècle connait, après une longue éclipse, un renouveau spectaculaire en Grande-Bretagne dans la première moitié du XVIII ${ }^{e}$ siècle. Si les convictions ariennes de Newton restent secrètes, ses amis anglicans William Whiston et Samuel Clarke, considéré comme le plus grand philosophe anglais du début du XVIII ${ }^{\mathrm{e}}$ siècle après Locke, les consignent dans des traités qui donnent lieu à de vives controverses (même si Clarke rejette l'accusation d'arianisme). Whiston et Clarke font des émules au sein de l'Église établie, mais l'arianisme se propage également au sein des Églises dissidentes. L'un de ses plus farouches partisans est sans conteste le ministre presbytérien Thomas Emlyn (bien qu'il préfère se définir comme unitarien). En revanche, cette hérésie est énergiquement combattue par le théologien anglican Daniel Waterland et par son homologue dissident Edmund Calamy, qui la pourfend dans treize sermons ${ }^{14}$.

5 Par ailleurs, en mettant l'accent sur le dogme de la prédestination, souvent absent des catéchismes ${ }^{15}$, Defoe exprime son attachement au calvinisme remis en cause par les disciples d'Arminius. Les dissidents comme les anglicans sont officiellement calvinistes, et nombre d'entre eux le sont effectivement. Toutefois, depuis le XVII siècle, l'arminianisme a gagné du terrain, non seulement au sein de l'Église anglicane, mais également des Églises dissidentes. Introduit dans la première sous William Laud et surtout présent, initialement, au sein de la "haute Église ", il compte également des partisans, au début du XVIII siècle, parmi les membres de la «basse Église » (low Church), à commencer par l'évêque de Bangor, Benjamin Hoadly. L'arminianisme rencontre aussi un certain succès auprès des dissidents. Certes, les indépendants, à l'instar de Stephen Lobb ou de John Guyse, restent le plus souvent fidèles au calvinisme. Cependant, dans le sillage de Richard Baxter qui, le premier, avait émis des réserves à l'égard de la doctrine calviniste de la prédestination, et dessiné une " voie moyenne ", nombreux sont les presbytériens de la génération suivante qui se tournent vers l'arminianisme. Tel est par exemple le cas de Daniel Williams ou de Samuel Chandler. L'orthodoxie calviniste conserve toutefois nombre de représentants, à l'image de l'exégète Henry Matthew ou de Daniel Wilcox qui, en 1716, se sépare de son assistant Henry Read, coupable d'avoir embrassé les thèses arminiennes ${ }^{16}$.

\section{Réquisitoires contre le catholicisme romain et le judaïsme}

6 Dans The New Family Instructor (1727), Defoe dénonce les croyances qu'il tient pour hétérodoxes de manière plus directe. Il commence par fustiger l'ennemi traditionnel, le catholicisme romain. L'une des stratégies qu'il utilise pour le discréditer consiste à remettre en cause l'autorité de son chef spirituel, le pape, et à contester sa suprématie. L'évêque de Rome ne doit son titre d'évêque universel qu'à un arrangement scélérat 
conclu en 590 entre Grégoire le Grand et le centurion romain Phocas. Ces deux hommes ont un ennemi commun, l'empereur Maurice, qui occupe la place convoitée par Phocas, et refuse d'octroyer le titre d'évêque universel à Grégoire. Phocas assassine Maurice et se proclame empereur à sa place. Grégoire reconnaît alors Phocas comme empereur légitime, et ce dernier, en contrepartie, confère le titre d'évêque universel à l'évêque de Rome - non pas à Grégoire lui-même, mais à son successeur, Boniface III. Le statut du pape en tant qu'évêque universel repose donc sur un assassinat et une usurpation, qui signent l'acte de naissance de l'église catholique romaine au détriment de la véritable Église chrétienne ${ }^{17}$. Il est donc vain d'invoquer une succession apostolique ininterrompue remontant à l'apôtre Pierre. Le siège papal est parfois resté vacant. À d'autres moments, plusieurs hommes l'ont occupé en même temps ${ }^{18}$. Par ailleurs, Defoe raille la prétention des papes à l'infaillibilité, et accuse nombre d'entre eux d'immoralité ${ }^{19}$. La liste de ces papes indignes est longue ; celle de leurs turpitudes l'est davantage encore. Nous nous contenterons ici de rapporter les propos de Defoe sur Alexandre VI (1492-1503): 'Alexander VI [...] was a Poisoner, a Murtherer, an Adulterer, insatiably Avaritious; and, as one Author, says of him, an Universal Villain' ${ }^{20}$. Pour discréditer le papisme, rien de tel que de mettre le doigt sur les errements des papes: ‘ Tis enough to make any body sick not of Popes only, but of Popery too' ${ }^{21}$.

7 De même, selon Defoe, certains saints catholiques ont mené une existence peu édifiante, à l'instar de saint Marc d'Aréthuse, coupable d'arianisme, de saint Thomas Becket, convaincu de rébellion, ou du régicide saint Jacques Clément ${ }^{22}$. D'autres ont été inventés de toutes pièces. Defoe se moque des saints catholiques, de leur exaltation, et des prétendus miracles qui leur sont attribués. Ses deux cibles préférées sont saint Ignace, fondateur de la Compagnie de Jésus, et saint François d'Assise, présenté comme un exalté qui se prend pour le Christ, notamment lorsqu'à l'image de ce dernier, il arbore cinq plaies. Seuls le Christ, ses apôtres et certains Pères de l'Église primitive ont accompli des miracles. Les vies des saints ont été enjolivées par des moines afin de fabriquer des légendes susceptibles d'impressionner les fidèles. Ces «légendes romaines " sont devenues synonymes de mensonges ${ }^{23}$. Par ailleurs, les saints ne doivent pas faire l'objet d'une quelconque vénération. Defoe dénonce leur culte, ainsi que celui de leurs images et de leurs reliques. Les prières d'intercession aux saints sont blasphématoires, y compris celles qui sont adressées à la Vierge Marie, puisque le seul médiateur entre l'homme et Dieu est le Christ ${ }^{24}$.

Defoe condamne en outre avec force la pratique des indulgences papales. Le pape n'a pas le pouvoir de pardonner nos péchés, et encore moins de les remettre à l'avance, ce qui est pratiquement une incitation à les commettre. Seul Dieu peut absoudre le pécheur ${ }^{25}$. D'après Defoe, le pape Léon $\mathrm{X}$ a vendu des indulgences pour subvenir aux besoins de ses enfants illégitimes, avant que Martin Luther ne dénonce cette supercherie ${ }^{26}$. Certains catholiques ont dépensé des sommes prodigieuses et se sont parfois séparés de leurs domaines pour acheter ces indulgences. D'autres apparemment inconscients du paradoxe de leur geste - ont volé afin de pouvoir en acquérir : 'It made Wives rob their Husbands, and Children their Fathers; nay, it made Fathers and Mothers strip their Families, for who would not rob and steal to buy themselves out of the Flames? ${ }^{27}$

9 À travers cette critique acerbe du catholicisme romain, Defoe rappelle certains des principaux traits de l'identité protestante : sa condamnation de la papauté, du culte des saints et des indulgences ne peut que rencontrer l'approbation de la grande majorité de 
ses lecteurs, qu'ils soient anglicans ou dissidents. Seuls les non-jureurs et les membres les plus intransigeants de la "haute Église ", à l'image de William Law ou d'Andrew Snape, sont susceptibles d'y trouver un motif d'insatisfaction, à savoir la remise en cause de la théorie de la succession apostolique ininterrompue des évêques, c'est-à-dire du droit divin de l'épiscopat, auquel Law, Snape et leurs amis sont particulièrement attachés ${ }^{28}$. Pour le reste, Defoe tient des propos très consensuels. Les arguments qu'il utilise font partie de la panoplie classique des propagandistes protestants, que ceux-ci appartiennent à l'Église établie ou aux Églises dissidentes. On les retrouve sous la plume d'auteurs aussi différents que le dissident presbytérien John Billingsley, l'anglican calviniste John Edwards, les anglicans modérés Isaac Barrow et William Fleetwood, ou l'ancien catholique récemment converti au protestantisme John Barville ${ }^{29}$. Defoe reprend nombre d'arguments utilisés par John Edwards dans The Doctrines Controverted between Papists and Protestants Particularly and Distinctly Consider'd: And Those which are Held by the Former Confuted (1724), en particulier ceux qui concernent la question de la suprématie et de l'infaillibilité papales, et celle des indulgences. Toutefois, il laisse de côté certains points abordés par Edwards tels que la doctrine de la transsubstantiation - qu'il ne fait que mentionner en passant -, le rôle de la messe, la confession auriculaire, ou encore le célibat du clergé. Ceci reflète la volonté de Defoe d'éviter à ce stade de son traité d'entrer dans des débats théologiques trop abscons, pour se concentrer sur les fondamentaux de l'anticatholicisme, plus susceptibles de fédérer ses lecteurs, notamment en s'attaquant à deux de leurs cibles préférées: les saints catholiques et les papes. S'agissant de ces derniers, l'anglican William Fleetwood avait montré l'exemple. Dans A Letter to the Reverend Dr. Snape (1718), il n'avait pas ménagé ses sarcasmes à l'égard de la papauté, produisant une liste commentée des "vice-gérants du Christ » depuis saint Pierre jusqu'à Léon X (1513-1521) censée prouver - non sans ironie - leur infaillibilité et leur exemplarité. En témoigne, par exemple, ce portrait de Jean XIII (965-972) :

John XIII [...] Was accused in a Synod before the Emperor Otho, for having put out the Eyes of some of his Cardinals, plucking out the Tongues of others, cutting off the Hands, Feet, Fingers, Noses, and Genitals of others: for ordaining Deacons (to perpetuate our Succession) in a Stable, for making Boys Bishops for Money, for ravishing both Virgins and Pilgrims, and lastly for calling upon the Devil for Assistance at Dice, and drinking to his Health. ${ }^{30}$

Le pape Séverin (638-640) n'est que l'exception qui confirme la règle : 'Severinus [...] was a pious charitable Man, but a bad Pope; for he not only did nothing to enlarge the Power of the Church, but even sacrilegiously permitted its Treasures to be diminished ${ }^{\prime 31}$. Le contenu de ces vignettes est proche de celui des portraits brossés par Defoe. Mais ce dernier s'abstient de tout humour pour mieux souligner la noirceur des papes et susciter l'indignation de ses lecteurs. C'est également leur indignation que Defoe cherche à provoquer lorsqu'il évoque le sordide accord entre Grégoire le Grand et Phocas qui débouche sur l'octroi de la suprématie papale. Sur ce point précis, les autres auteurs protestants proposent toutefois une version différente de celle de Defoe. L'accord concernant la suprématie romaine n'aurait pas été conclu entre Grégoire et Phocas, mais entre ce dernier et Boniface III. De plus, Boniface III n'aurait fait que reconnaître l'accession de Phocas à la tête de l'Empire consécutive à l'assassinat de Maurice; il n'y aurait pas eu de complot entre les deux hommes ${ }^{32}$. Mais peu importe ici l'exactitude historique : l'essentiel pour Defoe est de susciter par sa rhétorique antipapiste une approbation qu'il pourra ensuite exploiter lorsqu'il abordera avec ses lecteurs des sujets moins consensuels. Après avoir 
concentré les feux de sa rhétorique sur l'ennemi traditionnel papiste, il s'applique beaucoup plus brièvement - à mettre en évidence les errements du judaïsme.

D'après Defoe, les juifs commettent l'erreur de ne pas se rendre compte que le Christ est le Messie qu'ils attendent. En cela, ils vont à l'encontre de la Bible, qui annonce clairement la venue du Messie à l'époque du Christ. Toutes les prophéties concordent sur ce point. Par conséquent, si les juifs nient que le Christ est le Messie, ils doivent aussi récuser toute autorité à leurs prophètes. Ils ont également tort lorsqu'ils considèrent que le Messie a différé son avènement en raison de leurs péchés, car Dieu, qui est omniscient, savait à l'avance comment ils se comporteraient ${ }^{33}$. À l'image du ministre anglican George Stanhope ou du non-jureur Charles Leslie, Defoe se livre à une critique exclusivement religieuse du judaïsme ${ }^{34}$. Il n'exprime aucun préjugé antisémite, à la différence, par exemple, de John Grindley, qui accuse les juifs d'égoïsme et de cupidité35.

\section{Le déisme et l'arianisme au pilori}

La cible suivante de Defoe dans sa croisade contre les croyances hétérodoxes est le déisme, qu'il associe au scepticisme et à la libre-pensée. Les déistes, explique-t-il, ne nient pas l'existence de Dieu. À leurs yeux, celui-ci est infiniment bon, clément et bienveillant; il n'éprouve aucun ressentiment, aucune colère; il ne punit pas, ne se venge pas; l'enfer n'existe pas. À cela, Defoe répond que si Dieu est infiniment bon, saint, et pur, il ne peut considérer le péché, qui est par nature corrompu et impur, sans ressentiment, ni déplaisir. Sinon, il ne serait pas juste ${ }^{36}$. Pour étayer sa position, Defoe se fonde sur l'Écriture, qui établit que l'impiété et l'iniquité provoquent le courroux de Dieu $^{37}$. Celle-ci rapporte d'ailleurs de nombreux actes de vengeance divine sur des pécheurs, dont la destruction de Jérusalem annoncée par le Christ ${ }^{38}$. Defoe ajoute que, même aujourd'hui, Dieu intervient souvent dans les affaires du monde pour punir ceux qui ont péché. Il en donne de nombreux exemples, dont le suivant: 'Here was one, the other Day, that affirming a Thing he knew to be false, wish'd he might drop down dead immediately if it was not true, and immediately he sunk down and died, having only Time to acknowledge that he had lyed, and that God was just ${ }^{39}$. Ainsi, aux déistes qui se targuent d'argumenter conformément à la raison, Defoe oppose, d'une part, la force de la Révélation et, d'autre part, la réalité des faits qui, à ses yeux, l'emporte sur la conformité formelle à la raison: 'To argue against Reason is unreasonable, but to argue against Fact is impracticable ${ }^{30}$. Contrairement à ce que prétendent les déistes, la Révélation est fondée sur des bases solides et inattaquables. L'Écriture porte le sceau de l'autorité divine :

It is GOD and not Man, who speaks throughout the whole Book, and who was the first true Author of it: One immediately sees the Stamp of his Divine Authority upon every Part of it; there is surprizing Majesty and Greatness in every Word; it captivates the Mind, and gives the Soul such an Elevation of Thought, as cannot be found in any other Writings. ${ }^{41}$

13 Trois raisons montrent que la Bible est bien la Parole de Dieu : la connaissance de Dieu qu'elle nous transmet; la connaissance de nous-mêmes qu'elle nous communique ; et surtout, l'accomplissement des prophéties. Dieu seul connaît l'avenir. C'est donc lui qui l'a révélé aux prophètes pour qu'ils informent le monde de ses desseins ${ }^{42}$. 

William Wollaston, qui menacent l'orthodoxie protestante, dans un esprit didactique plutôt que polémique. Il ne s'agit pas pour lui de faire assaut d'érudition ou de porter l'estocade à tel ou tel polémiste rival, mais de montrer comment le déisme bafoue certains grands principes du protestantisme, et de prouver leur bien-fondé. En réaffirmant le rôle de la Providence, le statut de la Bible comme réceptacle de la parole divine, ou l'exactitude des prophéties, il défend certaines de ses convictions les plus profondément ancrées, qui irriguent d'ailleurs nombre des œuvres qu'il compose au cours des années 1720 . Pour contrecarrer le rationalisme des déistes, Defoe s'appuie à la fois sur la Révélation et sur l'expérience. Il prend ses distances avec la raison, dont il s'était naguère réclamé pour combattre «l'enthousiasme » de certains sectateurs. Mais la donne a changé, puisque la notion d'enthousiasme a été redéfinie par les déistes et autres rationalistes pour stigmatiser les tenants inflexibles de l'orthodoxie protestante. En pourfendant le déisme, Defoe fait cause commune avec des protestants de sensibilités très différentes, puisque ceux-ci incluent notamment les ministres dissidents Theophilus Lobb et Isaac Watts, le recteur anglican Benjamin Bayly, et le flamboyant polémiste non-jureur Charles Leslie, qui réaffirme avec force l'autorité divine de l'Écriture dans A Short and Easie Method with the Deists $(1698)^{43}$.

es catholiques romains, les juifs et les déistes, Defoe, dans sa campagne contre les croyances hétérodoxes, a une dernière cible: l'antitrinitarisme, en particulier l'arianisme. Contrairement aux déistes, les ariens, les sociniens et autres antitrinitariens ne nient pas l'autorité divine des Écritures, mais ils en détournent le sens par leurs interprétations erronées. Face à ces errements exégétiques, Defoe affirme la divinité du Christ et sa consubstantialité avec le Père. Le Christ, explique-t-il, se présente lui-même comme le Fils de Dieu dans deux passages de l'évangile selon saint Jean (Jean 5:18, Jean 10:33). Or, contrairement à ce que prétendent les ariens, être le Fils de Dieu revient à être soi-même Dieu. Le Père et le Fils ne portent-ils pas tous les deux le nom de Jéhovah? Les termes «Dieu» et «Fils de Dieu» n'impliquent pas de subordination du second au premier. D'ailleurs, lorsqu'il affirme que son Père et lui « ne font qu'un » (Jean 10:30), le Christ indique que son Père et lui sont égaux. Ils ont la même essence et la même substance. Certes, le Christ déclare lors de la Passion que son père est « plus grand que [lui] » (Jean 10:29, Jean 14:28), mais il s'exprime alors en tant que médiateur, pendant son humiliation. La subordination dont il est question à ce moment-là concerne sa fonction, et non sa personne. Ceci reflète la double nature du Christ : à la fois divine et humaine. Le Christ, dans sa nature divine, a existé de toute éternité avec le Père et le Saint-Esprit ${ }^{44}$. Defoe réaffirme avec force le dogme de la Trinité. À l'appui de cette doctrine, il invoque un passage controversé de la première épître de saint Jean : "Car il y en a trois dans le Ciel qui rendent témoignage: le Père, la Parole, et le Saint-Esprit; et ces trois-là ne sont qu'un" (1 Jean 5:7). Defoe reconnaît que l'authenticité de ce verset est contestée par certains exégètes. Mais il la défend avec fermeté, et remet en cause la sincérité de ceux qui la récusent. En tout état de cause, il observe que le dogme de la Trinité est attesté par d'autres passages de l'Écriture, en particulier Jean 10:33

16 Les arguments de Defoe sont adaptés aux sociniens et aux ariens, mais ils visent sans doute davantage les seconds, dans la mesure où il ne se borne pas à démontrer la divinité du Christ (que contestent les sociniens), mais s'applique également à prouver que le Père et le Fils sont égaux et consubstantiels (ce que nient les ariens). De fait, les 
écrits de Thomas Emlyn, comme ceux de William Whiston et de Samuel Clarke, trouvent un écho favorable au sein de l'Académie dissidente d'Exeter où, au printemps 1718, l'arianisme se répand comme une traînée de poudre. Des étudiants et plusieurs ministres, dont le presbytérien James Pierce, embrassent l'hérésie. Sommés de souscrire à une déclaration condamnant l'arianisme, les ministres incriminés refusent de s'exécuter. L'affaire est portée à la connaissance des ministres dissidents londoniens, qui se réunissent à Salters's Hall en février 1719 pour en débattre. À cette occasion, une courte majorité des ministres présents estime qu'il est inutile de demander à leurs collègues d'Exeter de souscrire à une déclaration en faveur de la Trinitée ${ }^{46}$. Les membres de cette majorité, composée en grande partie de ministres presbytériens, expliquent que leur vote négatif ne vise pas le contenu de la déclaration, mais le fait de se la voir imposer. Toutefois, dans un pamphlet intitulé Some Remarks upon the Late Differences among the Dissenting Ministers and Preachers (1719), Defoe exprime des doutes quant à leur bonne foi. Il regrette que les partisans de la déclaration en faveur de la Trinité ne l'aient pas emporté, et accuse James Pierce de promouvoir l'arianisme ${ }^{47}$. Defoe condamne en outre cette hérésie dans The Political History of the Devil (1726), reprochant notamment à Milton de l'avoir introduite au cœur de l'intrigue de Paradise Lost :

The Plot is wrong laid [...] because contradicted by the Scripture account, according to which Christ was declared in Heaven, not then, but from Eternity, and not declared with power, but on Earth [...] so that Mr. Milton is not orthodox in this part, but lays an avow'd foundation for the corrupt Doctrine of Arius, which says, there was a time when Christ was not the Son of GOD. ${ }^{48}$

Dans A New Family Instructor, Defoe rejette méthodiquement les différents arguments des ariens, et se fait pédagogue pour rendre accessible à un large public le débat complexe sur la Trinité et les thèses de Daniel Waterland et d'Edmund Calamy qui la défendent ${ }^{49}$, même s'il fait occasionnellement montre de son érudition, en particulier lorsque, dans la lignée de David Martin, théologien protestant français réfugié à Utrecht $^{50}$, il s'efforce d'établir l'authenticité de 1 Jean 5:7 contestée par Thomas Emlyn $^{51}$.

18 Ainsi, dans sa défense de l'orthodoxie protestante, Defoe justifie l'existence de Dieu (contre les athées), l'autorité divine de la Bible (contre les déistes), le dogme de la Trinité (contre les sociniens et, surtout, les ariens), et la doctrine de la prédestination (contre les arminiens). En ce qui concerne ces deux derniers points, sa position est loin d'être consensuelle parmi les dissidents. Elle l'est encore moins parmi les presbytériens, puisque c'est parmi eux que l'arminianisme et l'arianisme sont le plus répandus.

\section{Héritier de Samuel Annesley}

Pour comprendre la position de Defoe, il est nécessaire de prendre en compte son éducation religieuse et la situation des presbytériens dans les années 1670. Au cours de cette décennie, ceux-ci se divisent en deux branches : les «Professeurs» (Dons) et les «Canetons» (Ducklings). Les premiers comptent dans leurs rangs des figures telles que Richard Baxter, William Bates ou John Howe, éminents théologiens censés être quelque peu condescendants à l'égard de leurs plus jeunes collègues, les "Canetons». Les positions défendues par les "Professeurs " sont modérées, plutôt conservatrices. À l'inverse, les "Canetons", dont les principaux représentants sont Samuel Annesley, Thomas Vincent et Thomas Watson, expriment des points de vue relativement 
radicaux, souvent proches de ceux des indépendants. Le principal contentieux entre les deux branches du presbytérianisme porte sur l'attitude à adopter face à l'Église d'Angleterre : les «Professeurs" sont favorables à une réunification (comprehension) avec l'Église anglicane, tandis que les «Canetons", qui y sont hostiles, aspirent à une tolérance, dont ils bénéficieraient au même titre que les indépendants. Toutefois, ces deux familles presbytériennes divergent également sur le plan du dogme. Les "Professeurs " prennent leurs distances par rapport à l'orthodoxie calviniste. Baxter défend une "voie moyenne "entre prédestination et libre arbitre, et nombre de ses successeurs opteront pour l'arminianisme, avant, dans certains cas, d'embrasser l'arianisme. En revanche, les "Canetons", à l'image des indépendants, s'inscrivent dans la tradition calviniste, et souscrivent à la doctrine de la prédestination; ils sont en outre très attachés à celle de la Trinitées. En tant que presbytérien, Defoe est directement concerné par le clivage entre «Professeurs » et «Canetons». Il se trouve que le pasteur de ses parents n'est autre que Samuel Annesley, principale figure des seconds. Par ailleurs, l'école où Defoe est envoyé, l'Académie de Charles Morton, à Newington Green, est essentiellement fréquentée par des "Canetons" (et par des indépendants) ${ }^{53}$. Ainsi, tout naturellement, Defoe embrasse les opinions de Samuel Annesley et de ses disciples: d'où son attachement à un calvinisme traditionnel, trinitaire et prédestinataire.

\section{Conclusion}

Après avoir été le porte-parole officieux des dissidents pendant plus d'une décennie, Defoe, vers le milieu des années 1710, s'érige en défenseur de l'orthodoxie protestante. Dans The Family Instructor, il expose sa vision du protestantisme, et défend les doctrines de la Trinité et de la prédestination face à la montée des croyances hétérodoxes. Dans The New Family Instructor, il s'attaque à ces dernières de manière plus directe. S'il commence par fustiger le catholicisme romain et le judaïsme, il dénonce ensuite avec force le déisme et l'antitrinitarisme sous ses différentes formes. Le principal objectif de Defoe est de faire pièce à l'arianisme. Alors même que cette hérésie n'épargne pas l'Église d'Angleterre, elle semble rencontrer un écho plus favorable encore au sein des Églises dissidentes, en particulier parmi ses coreligionnaires presbytériens, comme le montrent l'affaire d'Exeter et la rencontre de Salters' Hall. Defoe est donc inquiet. À juste titre: dans les années qui suivront, l'arianisme gagnera du terrain parmi les dissidents, qui, pour certains d'entre eux, évolueront ensuite vers l'unitarisme.

Dans la préface de ses treize sermons sur la Trinité, Edmund Calamy livre l'une des raisons pour lesquelles il a combattu l'arianisme : 'I am in hope that these Discourses, together with a good Number of Tracts lately publish'd, may help to prevent its being hereafter said, that the Dissenters did not at this Time appear against Arianism, when it so much threaten'd us ${ }^{\prime 54}$. À l'instar de Calamy, Defoe peut se targuer d'avoir fait tout ce qui était en son pouvoir pour maintenir les dissidents dans le giron de l'orthodoxie protestante. 


\section{NOTES}

1. VOLTAIRE, Lettres philosophiques [1734], Paris : Garnier-Flammarion, 1964, p. 42.

2. John Robert MORE, 'Defoe's Religious Sect', The Review of English Studies, vol. 17, $\mathrm{n}^{\circ}$ 68, octobre 1941, pp. 461-467.

3. Voir, par exemple, Daniel DEFOE, An Enquiry into Occasional Conformity. Shewing that the Dissenters are no Way Concern'd in it, London, 1702, pp. 30-31.

4. Daniel DEFOE, The Family Instructor in Three Parts; I. Relating to Fathers and Children. II. To Masters and Servants. III. To Husbands and Wives, $2^{\text {nd }}$ ed., London, 1715, p. 3.

5. Maurice WILES, Archetypal Heresy: Arianism through the Centuries, Oxford: Oxford University Press, 1996, p. 135. L'arianisme, ou doctrine d'Arius (256-336), remet en cause le dogme de la Trinité en postulant que le Fils n'est pas de même nature que le Père et que le premier est subordonné au second. Toutefois, à la différence du socinianisme, cette doctrine ne nie pas toute divinité au Fils.

6. Ce processus est examiné dans Russel E. RICHEY, 'From Puritanism to Unitarianism in England: A Study in Candour', Journal of the American Academy of Religion, vol. 41, $\mathrm{n}^{\circ}$ 3, septembre 1973, pp. 371-385.

7. DEFOE, Family Instructor, p. 18.

8. Ibid., pp. 21-23.

9. Ibid., p. 27.

10. Ibid., p. 21.

11. Ibid., p. 28.

12. Ibid., pp. 23-24.

13. Ibid., pp. 70, 225, 228, 260, 282.

14. WILES, op. cit., pp. 62-164.

15. Ian GREEN, The Christian's ABC: Catechisms and Catechizing in England c. 1530 - 1740, Oxford: Clarendon Press, 1996, pp. 350-386.

16. C. Gordon BOLAM, Jeremy GORING et al., The English Presbyterians: From Elizabethan Puritanism to Modern Unitarianism, London: George Allen and Unwin Ltd, 1968, pp. 103-104, 109-110, 117-120, 125, 127, 135-137, 150-155, 166, 168, 180-181.

17. Daniel DEFOE, A New Family Instructor; in Familiar Discourses between a Father and his Children, on the most Essential Points of the Christian Religion, London, 1727, pp. 48-49, 65, 68-73.

18. Ibid., pp. 93, 127-130. Lors du Grand Schisme d'Occident (1378-1417), deux lignées de papes se disputent la direction de l'Église catholique, l'une établie à Rome, l'autre en Avignon. Urbain VI (1378-1389), Boniface IX (1389-1404), Innocent VII (1404-1406) et Grégoire XII (1406-1415) appartiennent à la première ; Clément VII (1378-1394) et Benoît XIII (1394-1417), à la seconde. À cela, il faut ajouter que, de 1409 à 1415, les papes Alexandre V (1409-1410) et Jean XXIII (1410-1415), issus du Concile de Pise (1409) revendiquent également le trône de Pierre. Selon la tradition catholique, seuls les papes de Rome sont légitimes; ceux d'Avignon et de Pise sont qualifiés d'antipapes. L'apparition d'antipapes n'est pas limitée à la période du Schisme d'Occident : on en recense une trentaine entre le III ${ }^{\mathrm{e}}$ siècle et le milieu du XV $\mathrm{XV}^{\mathrm{e}}$ siècle.

19. Ibid., pp. 46, 60, 71, 97, 112, 136-137, 148, 351.

20. Ibid., p. 136.

21. Ibid., p. 137.

22. En fait, Jacques Clément (1567-1589), moine dominicain ligueur qui assassine le roi Henri III (et non Henri IV, comme l'écrit Defoe) le $1^{\text {er }}$ août 1589 , n'a pas le rang de saint, bien que le pape Sixte $\mathrm{V}$ ait songé à canoniser ce religieux qui, par son geste, avait vengé aux yeux de certains catholiques l'assassinat du duc de Guise l'année précédente. 
23. DEFOE, New Family Instructor, pp. 56-57, 166, 176-182, 187-190, 209.

24. Ibid., pp. 150-151, 155, 160, 162, 186, 222, 228-229.

25. Ibid., pp. 198, 226, 241.

26. Ibid., p. 202.

27. Ibid., p. 205.

28. Voir, par exemple, Andrew SNAPE, A Letter to the Bishop of Bangor, Occasion'd by his Lordship's Sermon Preach'd before the King at St. James's, March 31 ${ }^{\text {st }}$, 1717, London, 1717, pp. 18-20; William LAW, The Bishop of Bangor's Late Sermon and his Letter to Dr. Snape in Defence of it, Answer'd, London, 1717, pp. 9-16.

29. Voir notamment John BILLINGSLEY, Sermons against Popery, London, 1723, pp. 281-296; John EDWARDS, The Doctrines Controverted between Papists and Protestants Particularly and Distinctly Consider'd: And Those which are Held by the Former Confuted, London, 1724; Isaac BARROW, 'A Treatise of the Pope's Supremacy', in The Works of Isaac Barrow, D. D., 3 vols., London, 1716, vol. 1, pp. 557-755; [William FLEETWOOD], A Letter to the Reverend Dr. Snape; Wherein the Authority of the Christian Priesthood is Maintain'd; the Uninterrupted Succession of Bishops from the Apostles Days is Lineally Deduced; and the Cavils of Hereticks and Fanaticks are Answer'd, $2^{\text {nd }}$ ed., London, 1718; [John BARVILLE], An Account of the Late Conversion of Mr. John Barville Alias Barton, from Popery to the Reformed Church of England, London, 1710.

30. [FLEETWOOD], op. cit., p. 36.

31. Ibid., p. 27.

32. Voir, par exemple, John HOWE, A Sermon Concerning Union among Protestants, Preached and Published About Twenty Years Ago, London, 1703, pp. 45-46; Gilbert BURNET, The Royal Martyr Lamented, in a Sermon Preached at the Savoy, on King Charles the Martyr's Day, 1675, London, 1710, pp. 4, 45; [Charles LESLIE], The Case Stated, Between the Church of Rome and the Church of England, London, 1713, pp. 107-108.

33. DEFOE, New Family Instructor, pp. 274-277, 307-310.

34. George STANHOPE, The Truth and Excellence of the Christian Religion Asserted: Against Jews, Infidels and Hereticks, London, 1702; [Charles LESLIE], A Short and Easie Method with the Jews, $3^{\text {rd }}$ ed., London, 1715.

35. John GRINDLEY, The Jews Generosity Explain'd, London, [1722], pp. 13, 15.

36. DEFOE, New Family Instructor, pp. 225-256.

37. Ibid., p. 256. Defoe renvoie son lecteur à Romains 1:13, mais il s'agit manifestement d'une coquille. En fait, le passage auquel il se réfère est Romains 1:18: "Car la colère de Dieu se révèle [pleinement] du Ciel sur toute impiété et injustice des hommes qui retiennent injustement la vérité captive »(Bible David Martin, 1744).

38. Ibid., pp. 258-259, 264, 265.

39. Ibid., p. 258.

40. Ibid., p. 257.

41. Ibid., p. 260.

42. Ibid., pp. 260-261, 264, 269, 272, 274.

43. Theophilus LOBB, A Discourse on Ministerial Instruction, London, 1712, pp. 40-62 ; Isaac WATTS, Caveat Against Infidelity, London, [1729], pp. 37-126; Benjamin BAYLAY, The Truth of the Christian Revelation, Prov'd from the Nature and Greatness of its Miracles; and of the Usefulness and Necessity of Creeds in General, in Opposition to Deists and Free-Thinkers, London, 1713; [Charles LESLIE], A Short and Easie Method with the Deists, London, 1698.

44. DEFOE, New Family Instructor, pp. 278-282, 288-290, 296-298.

45. Ibid., pp. 281, 351-368.

46. BOLAM, GORING et al., op. cit., pp.151-174; WILES, op. cit., pp. 137-142.

47. Maximilian NOVAK, Daniel Defoe: Master of Fictions, Oxford: Oxford University Press, 2001, pp. 523-524.

Revue Française de Civilisation Britannique, XVIII-1 | 2013 
48. Daniel DEFOE, The Political History of the Devil [1726], New York: AMS Press, 2003, p. 57.

49. Voir, par exemple, Daniel WATERLAND, A Vindication of Christ's Divinity, Cambridge, 1719; Edmund CALAMY, Thirteen Sermons Concerning the Doctrine of the Trinity, London, 1722.

50. Initialement publiés en français, les différents ouvrages de David Martin visant à démontrer l'authenticité de 1 Jean 5:7 bénéficient d'une traduction anglaise, à l'instar de David MARTIN, $A$ Critical Dissertation upon the Seventh Verse of the Fifth Chapter of St. John's first Epistle, There are Three, that Bear record in Heaven, \&c., London, 1719.

51. Thomas EMLYN, 'An Inquiry into the Original Authority of 1 John 5:7', in Thomas EMLYN, A Collection of Tracts, Relating to the Deity, Worship and Satisfaction of the Lord Jesus Christ, London, 1719, pp. 305-356.

52. BOLAM, GORING et al., op. cit., pp. 87, 95-105, 119-120.

53. Katherine CLARK, Daniel Defoe: The Whole Frame of Nature, Time and Providence, New York: Palgrave Macmillan, 2007, pp. 39-40.

54. CALAMY, op. cit, 'The Preface'.

\section{RÉSUMÉS}

Après avoir été pendant de longues années le porte-parole officieux de ses coreligionnaires dissidents, Defoe, dans The Family Instructor (1715-1718) et dans The New Family Instructor (1727), s'exprime au nom de tous les protestants. Il s'agit de défendre l'orthodoxie protestante contestée non seulement, comme elle l'était naguère, par le catholicisme et le judaïsme, mais surtout par des doctrines hétérodoxes telles que le déisme, le socinianisme et l'arianisme, qui ont le vent en poupe au cours des premières décennies du XVIII ${ }^{\mathrm{e}}$ siècle. Pour les discréditer, Defoe emprunte des arguments aux polémistes anglicans comme aux propagandistes dissidents. Il défend avec une vigueur particulière les dogmes de la Trinité et de la prédestination, alors que nombre de ses coreligionnaires presbytériens inclinent à l'arminianisme et à l'arianisme. Cet article montre que l'attachement de Defoe à ces dogmes peut s'expliquer par son appartenance à l'aile la plus stricte du presbytérianisme jadis incarnée par Samuel Annesley (1620-1696).

After being for many years the officious spokesman for Dissent, Defoe, in The Family Instructor (1715-1718) and The New Family Instructor (1727), speaks in the name of all English Protestants. His aim in these works is to defend Protestant orthodoxy, which was being challenged not only, as it had been for ages, by Roman Catholicism and Judaism, but by heterodox beliefs such as Deism, Socinianism and Arianism, which were in the ascendant in the first decades of the eighteenth century. In order to discredit these beliefs, Defoe borrows arguments from both Anglican polemicists and Dissenting propagandists. He defends with a special determination the dogmas of the Trinity and predestination, at a time when many of his Presbyterian coreligionists were inclining towards Arminianism and Arianism. This essay argues that Defoe's attachment to these dogmas can be accounted for by his early exposure to the strict Presbyterianism of Samuel Annesley (1620-1696). 
AUTEUR

YANNICK DESCHAMPS

Université Paris-Est Créteil 